\title{
Identificación de sitios de encuentro de parejas sexuales en dos ciudades de la frontera sur de México, mediante el método PLACE
}

\author{
Mirka N egroni-Belén, Lic en Psicol, M en Admon Púb, ${ }^{(1)}$ Galileo Vargas-Guadarrama, MC, (1) \\ Celina Magally Rueda-N eria, Lic en Inf Admin, ${ }^{(1)}$ Sarah Bassett-Hileman, M en SP, ${ }^{(2)}$ Sharon W eir, PhD, ${ }^{(2)}$ \\ Mario Bronfman, Lic en Sociol, Dr en SP.(1)
}

\begin{abstract}
Negroni-Belén M,Vargas-Guadarrama G, Rueda-Neria CM, Bassett-Hileman S, Weir S, Bronfman M. Identificación de sitios de encuentro de parejas sexuales en dos ciudades de la frontera sur de México, mediante el método PLACE Salud Publica Mex 2003;45 supl 5:S647-S656. El texto completo en inglés de este artículo también está disponible en: http://www.insp.mx/salud/index.html

\section{Resumen}

Objetivo. Identificar sitios de encuentro de nuevas parejas sexuales en dos ciudades de la frontera sur de México. Material y métodos. En un estudio epidemiológico descriptivo se encuestó, en mayo de 2001, a informantes clave de la comunidad en dos ciudades fronterizas del sur de México para identificar sitios de encuentro de nuevas parejas. Se visitó cada sitio reportado y se encuestó a una persona del lugar. De una submuestra de sitios, se encuestó a personas que socializaban en los mismos. Resultados. Los informantes clave de la comunidad reportaron 134 sitios en Chetumal y 111 en Ciudad Hidalgo. Se obtuvo información de las car acterísticas de 89 sitios en Chetumal y 42 en Ciudad Hidalgo, ambos con escasas actividades preventivas delVIH/ SIDA. Cerca de $21 \%$ de usuarios encuestados reportaron haber conocido una nueva pareja en los sitios en las últimas cuatro semanas. Conclusiones. Son necesarias acciones de prevención en los sitios de encuentro de nuevas parejas sexuales. Este método puede proveer información para planificar futuras intervenciones. El texto completo en inglés de este artículo también está disponible en: http:// www.insp.mx/salud/index.html
\end{abstract}

Palabras clave: prevención; VIH/SIDA; infecciones de transmisión sexual; México

\author{
Negroni-Belén M, Vargas-Guadarrama G, \\ Rueda-Neria CM, Bassett-Hileman S, \\ Weir S, Bronfman M. \\ Identification of meeting sites of new sexual \\ partners in two Southern border cities in Mexico \\ using the PLACE method. \\ Salud Publica Mex 2003;45 suppl 5:S647-S656. \\ The English version of this paper is available too at: \\ http://www.insp.mx/salud/index.html
}

\begin{abstract}
A bstract
Objective. To identify the meeting sites of new sexual partners in two Southern border cities in Mexico. Material and Methods A descriptive epidemio logic study was carried out in May 2001, by surveying key informants in two border cities. Each reported site was visited to interview a resident subject. Subjects socializing in a subsample of sites were also interviewed. Results. The key informants of the community reported 134 meeting sites in Chetumal and 111 in Ciudad H idalgo. Both sites had scarce HIV/A ID S prevention activities. Characteristics were obtained for 89 sites in Chetumal and 42 in Ciudad Hidalgo.A Imost $21 \%$ of interviewees reportedly met a new sexual partner in these sites in the past four weeks. Conclusions. Preventive actions are needed in meeting sites of new sexual partners. This method may provide information to plan for future interventions. The English version of this paper is available too at:http://www.insp.mx/salud/index.html
\end{abstract}

Key words: prevention; HIV/AIDS; sexually transmitted diseases; Mexico

(1) Centro de Investigación en Sistemas de Salud. Instituto Nacional de Salud Pública. Cuernavaca, Morelos, México.

(2) MEASURE Evaluation, Carolina Population Center, University of N orth Carolina at Chapel Hill, N orth Carolina, United States.

Fecha de recibido: 11 de diciembre de 2002 • Fecha de aprobado: 25 de julio de 2003

Solicitud de sobretiros: Mirka N egroni. Instituto N acional de Salud Pública, Centro de Investigación en Sistemas de Salud.

Avenida Universidad 655, colonia Santa María A huacatitlán 62508 Cuernavaca, Morelos, México

E-mail: mnegroni@ correo.insp.mx 
Los recursos para programas de prevención de la infección por el virus de inmunodeficiencia humana $(\mathrm{VIH})$, así como el monitoreo y evaluación de las intervenciones, son extremadamente limitados, razón por la cual surge la necesidad de enfocar las acciones preventivas donde sean más costo efectivas. ${ }^{1}$ Una barrera en el desarrollo de intervenciones basadas en información obtenida de las redes ha sido la falta de métodos válidos, rápidos y confiables para describir y analizar una red dinámica de formación de nuevas parejas sexuales en una población definida, de tal forma que sea útil para la planeación de una intervención. Las áreas con mayor incidencia de infección por el VIH se han llamado áreas de alta transmisión (AAT). ${ }^{2}$

El método Priorities for Local AIDS Control Efforts (PLACE) es una herramienta nueva para identificar de manera rápida $\mathrm{AAT}$ y aquellos sitios específicos dentro de esas áreas donde deben ser enfocados los programas preventivos de infecciones de transmisión sexual (ITS) y VIH. El método fue desarrollado con base en modelos epidemiológicos del curso de la epidemia del VIH y datos empíricos que muestran agrupamientos geográficos de ITS en las comunidades. Se basa en el concepto de grupo medular, introducido por Yorke et al en 1978; ${ }^{2}$ los modelos matemáticos ${ }^{3}$ desarrollados por Anderson y May, y el reciente modelo de fase específica ${ }^{4}$ descrito por Wasserheit y Aral, los que resaltan la importancia del patrón de redes sexuales y la frecuencia de adquisición de una nueva pareja sexual en una comunidad. Este modelo sostiene que las estrategias óptimas de control de las ITS requieren dos tipos de aproximación para el crecimiento y para el mantenimiento de las redes. Existe una necesidad urgente de intervenir con acciones preventivas en los sitios en donde se dé una alta frecuencia de formación de nuevas parejas sexuales, ya que esto influye en el mantenimiento o diseminación de una epidemia del VIH.

La metodología PLACE se basa en los siguientes principios y suposiciones:

1) Los programas preventivos de la infección por $\mathrm{VIH} / \mathrm{SIDA}$ deben enfocarse en lugares donde se encuentran las personas con alta frecuencia de formación de parejas sexuales

2) Los datos contextuales, demográficos y epidemiológicos disponibles pueden proveer información para identificar las áreas con mayor probabilidad de tener patrones de formación de nuevas parejas capaces de diseminar y mantener la infección. Dentro de las AAT el objetivo es conocer los sitios donde las personas conocen a nuevas parejas sexuales

3) El método no depende del autorreporte de comportamiento, del rastreo de contactos o de mencionar los nombres de las parejas sexuales, lo cual minimiza el sesgo; no se requiere de pruebas serológicas del $\mathrm{VIH}$. El método se desarrolla en un periodo corto y no requiere la participación de expertos o técnicos de manera externa. La metodología puede proveer indicadores útiles para el monitoreo y evaluación de futuras intervenciones.

Este método fue desarrollado en la Universidad de Carolina del Norte (UCN) y piloteado en 1999 en Ciudad del Cabo, Sudáfrica. Desde entonces se han realizado adaptaciones para la aplicación del estudio en Burkina Faso, Uganda, Tanzania y Rusia. ${ }^{5}$ En México, el Instituto Nacional de Salud Pública (INSP) adaptó y realizó el estudio utilizando la metodología PLACE en dos ciudades diferentes de la frontera sur de México en el marco del proyecto de Poblaciones Móviles y VIH/SIDA en Centroamérica, México y Estados Unidos en 2001. ${ }^{6}$ Dichas ciudades fueron seleccionadas por el Centro Nacional para la Prevención del VIH/SIDA y otras Infecciones de Transmisión Sexual (CENSIDA). En este trabajo se presentan los resultados del estudio como una referencia para la planeación estratégica en VIH/SIDA en la Región.

\section{Material y métodos}

Estudio epidemiológico descriptivo en el cual se encuestó, en mayo de 2001, a informantes clave de la comunidad (ICC) en dos ciudades fronterizas del sur de México para identificar sitios de encuentro de nuevas parejas sexuales. El estudio se realizó en un periodo de cuatro semanas. En el estado de Chiapas se seleccionó a Ciudad Hidalgo por presentar un alto flujo de población móvil de Centroamérica hacia los Estados Unidos de América. En Quintana Roo se eligió a la ciudad de Chetumal por ser la capital del estado; su frontera con Belice presenta un flujo importante de población móvil, tanto de Belice como de otros países de Centroamérica. ${ }^{7}$ En esta ciudad se encuentran bases militares y de la marina mexicana cuyos miembros activos se relacionan con la población local. De ambas ciudades seleccionadas se obtuvo información contextual a través de los estudios etnográficos ${ }^{* * *}$

\footnotetext{
* Bronfman M, Leyva R. Migración y SIDAen México y Centroamérica. Seminario-Taller de Cooperación México-Centroamérica sobre prevención y control de ITS/VIH/SIDA con especial atención en las poblaciones móviles; Mimeo. 24 mayo 1999. Tuxtla Gutiérrez, Chiapas, México.

**Hernández-Rosete D. Notas para una etnografía de Chetumal Quintana Roo. Migración y SIDA en Centroamérica, México y Estados Unidos de Norteamérica. Instituto Nacional de Salud Pública, Cuernavaca, Morelos: 2001. Mimeo

${ }^{* * *}$ Guerrero C. Reporte de observación etnográfica. Ciudad Hidalgo: donde la vida no vale nada. Migración y SIDA en Centroamérica, México y Estados Unidos de Norteamérica. Mimeo. Cuernavaca, Morelos: Instituto Nacional de Salud Pública, 2001.
} 
realizados por el INSP en el proyecto de Poblaciones Móviles y VIH/SIDA, los cuales permiten ver diferencias importantes en el flujo de poblaciones móviles y de actividades económicas. Otros datos contextuales que permiten ver las diferencias entre las ciudades se muestran en el cuadro I. En todas las etapas del estudio cuando se encuestó a una persona, siempre se obtuvo el consentimiento informado antes del inicio de cada encuesta. Las respuestas fueron anónimas. Se realizó doble captura de la información obtenida, usando un programa de manejo de bases de datos.

\section{Encuestas a informantes clave de la comunidad (ICC) para la identificación de los sitios}

El objetivo fue identificar todos los sitios posibles donde las personas residentes o que están de paso en las áreas designadas acuden para conocer a nuevas parejas sexuales. Se consideró como un informante clave de la comunidad a la persona que conocía el área y los sitios de encuentro de parejas sexuales, o poseía información sobre poblaciones móviles. Los taxistas, trici-

\section{Cuadro I}

Datos contextuales de las ciudades seleccionadas. México, 2001

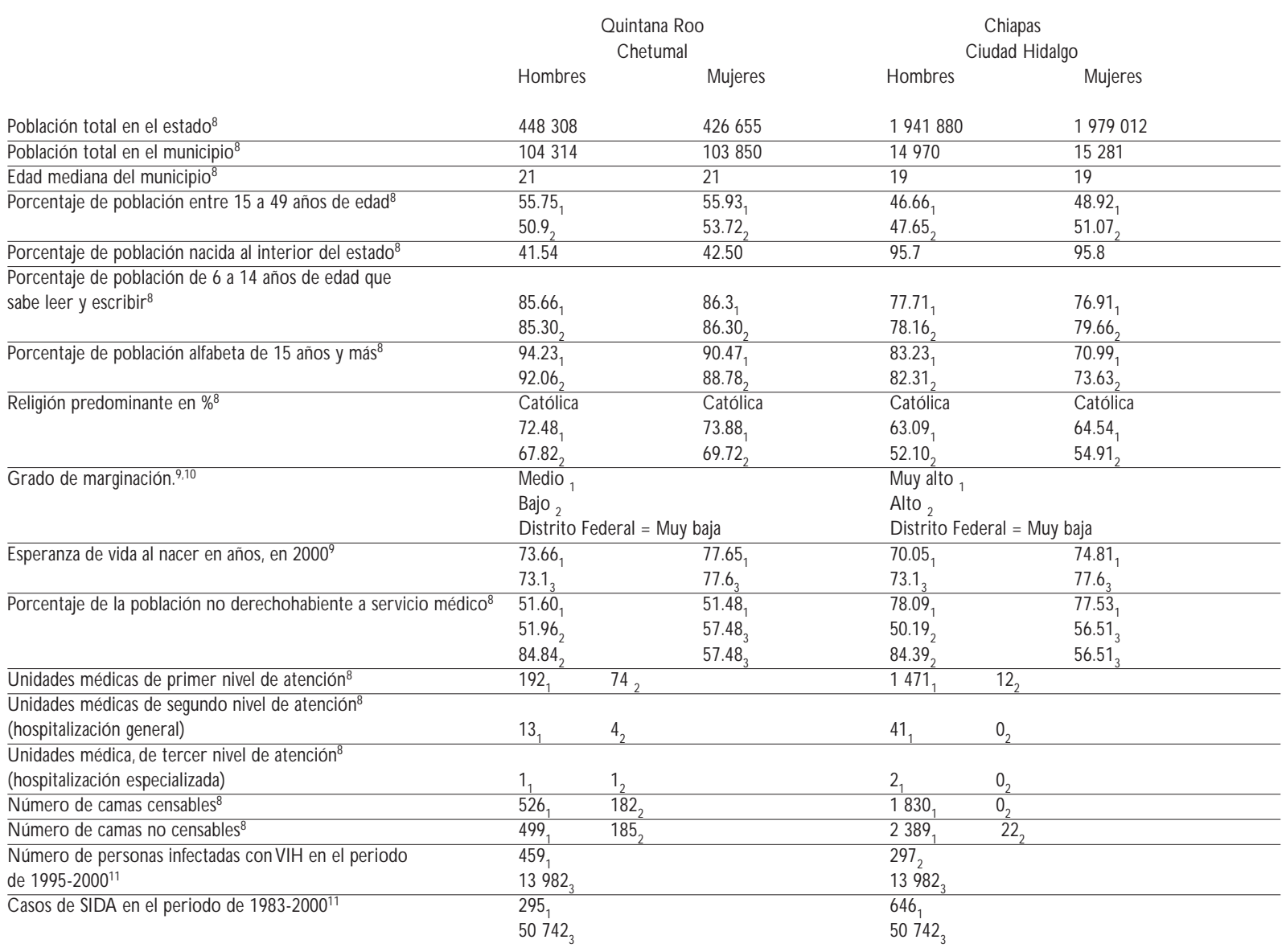

(1) Datos de referencia al estado

(2) Datos de referencia al municipio

(3) Datos de referencia a escala nacional 
cleros, traileros, agentes aduanales, de migración, policías, estudiantes, comerciantes, vendedores ambulantes, militares y trabajadores agrícolas fueron considerados informantes claves de la comunidad. Los ICC fueron seleccionados a conveniencia. Se encuestó a ICC en sitios como estaciones de autobuses, estaciones fronterizas, parques y plazas públicas, frecuentados por las poblaciones móviles. Se obtuvo información respecto al nombre, dirección y tipo de sitios en donde las personas buscan nuevas parejas sexuales. Los datos obtenidos por la encuesta se procesaron en campo con una hoja de cálculo.

\section{Visita y caracterización de cada sitio}

En esta etapa, los encuestadores visitaron cada sitio identificado por un ICC para verificar su existencia y encuestar a una persona conocedora del lugar o informante clave del sitio (ICS). La persona conocedora pudo ser el dueño, el gerente o un empleado (en el caso de bares/ cantinas, restaurantes, discotecas, botaneras y estaciones de autobuses), dueños u operadores del transporte (en el caso de taxistas, tricicleros, trabajadores del transporte de carga), comerciantes (en el caso de comercio formal e informal), policías, militares y otros empleados de gobierno (en el caso de las garitas fronterizas, estaciones aduanales, policías en las calles) y otras personas (en el caso de calles, parques y plazas públicas, escuelas, campos deportivos, etcétera). La encuesta se estructuró con las siguientes secciones: características del sitio, edad y sexo del encuestado, principales actividades dentro de los sitios, formación de parejas sexuales en el sitio, tipo de usuarios y actividades de prevención del VIH/SIDA/ ITS. La finalidad fue obtener de cada localidad una lista con información sobre las características de importancia de los sitios y confirmar parcialmente la información obtenida de los reportes de los ICC; se incluyeron aquellos sitios localizados en funcionamiento y en los que el encargado o dueño estuvo de acuerdo en que el equipo regresara a encuestar a los usuarios del lugar.

\section{Encuestas a las personas que interactuaban en los sitios}

Contar con la lista actualizada de sitios permitió que, las encuestas a usuarios o personas que socializaban se realizaran en una primera muestra de los lugares más frecuentemente reportados por los ICC; se eligió otro grupo de sitios mediante una selección aleatoria del resto de la lista de los reportados. Se buscó asistir a los seleccionados en la hora con más presencia de usuarios. Algunas de las personas que estaban interactuando en los sitios seleccionados fueron encuestadas con el propósi- to de obtener información respecto a la frecuencia de formación de nuevas parejas sexuales, características de su movilidad territorial, información sobre ITS/VIH/ SIDA, etcétera, y para confirmar los reportes de los ICC y de las encuestas con los ICS. La encuesta se estructuró con las siguientes secciones: datos sociodemográficos del encuestado, uso de condón, adquisición y formación de nuevas parejas sexuales, información recibida sobre ITS/VIH/SIDA, búsqueda de atención médica para tratamiento de ITS. Se encuestó un máximo de 24 personas (16 hombres y 8 mujeres) en cada sitio. $\mathrm{Al}$ interior de los sitios los individuos fueron seleccionados aleatoriamente por los encuestadores. El análisis estadístico se realizó por dos equipos diferentes (INSP y UCN) con el paquete de análisis estadístico STATA. La metodología del estudio se resume en el cuadro II.

\section{Resultados}

\section{Encuestas a informantes claves de la comunidad}

En Chetumal, en cuatro días se encuestó a 357 ICC. Sólo $7(<2 \%)$ rechazaron participar y otros seis fueron menores de edad, de lo cual se obtuvo un total de 344 reportes. Dos terceras partes se encontraban entre los 20 y 39 años de edad; el promedio de edad fue de 31 años. El $33 \%$ fueron mujeres. Los informantes nombraron un total de 176 sitios diferentes, 134 dentro del área del estudio. El 12.3\% de los informantes fueron taxistas, traileros o tricicleros; $7.5 \%$ dueño, gerente o empleado de un bar/cantina o restaurante; trabajadoras del sexo comercial (TSC) $0.3 \%$; comerciantes $18.6 \%$; guardia, militar, policía o marino $21.7 \%$; una persona que socializaba en sitios como parques, plazas, eventos públicos, $11.7 \%$, y otros informantes $27.9 \%$.

En Ciudad Hidalgo, en cuatro días se encuestó a 222 informantes, de los cuales $22(10 \%)$ rechazaron participar y $5(2.2 \%)$ fueron menores de edad, y se obtuvo un total de 195 reportes. Del total, $64 \%$ se encontraba entre los 20 y 39 años de edad; el promedio de edad fue de 34.4 años. El 28.6\% fueron mujeres. El tipo de informantes encuestados fue similar al de Chetumal, sin embargo se encuestó a más TSC. Los informantes nombraron un total de 111 sitios, 65 de los cuales fueron ubicados en el área de estudio. El $23.3 \%$ de los informantes fueron taxistas, traileros o tricicleros; $1.1 \%$ dueño, gerente o empleado de un bar/cantina o restaurante; $2.8 \%$ TSC; comerciantes $33.9 \%$; un guardia, militar, policía o marino $7.8 \%$; $17.2 \%$ algún individuo que socializaba en sitios como parques, plazas, evento públicos, y otros informantes $13.9 \%$. 


\section{Cuadro II Resumen del método PLACE}

\begin{tabular}{|c|c|c|c|}
\hline Etapa & O bjetivos & Métodos & Resultados \\
\hline 1 & $\begin{array}{l}\text { Identificar áreas con probables altas tasas de } \\
\text { incidencia de ITS/VIH y obtener información } \\
\text { contextual importante } \\
\text { Adaptación del protocolo, obtención de aprobación } \\
\text { ética y apoyo comunitario }\end{array}$ & $\begin{array}{l}\text { Síntesis de información y reportes epidemiológicos, } \\
\text { datos censales, reportes de salud, mapas e información } \\
\text { contextual.Traducción del protocolo, adaptación al } \\
\text { contexto y lenguaje, obtención de aprobación ética } \\
\text { por el comité del Instituto N acional de Salud Pública }\end{array}$ & $\begin{array}{l}\text { Selección de dos ciudades fronterizas al sur de México. } \\
\text { A probación del protocolo adaptado incluyendo la } \\
\text { metodología, método de muestreo, formatos de } \\
\text { recolección de datos, manual de los encuestadores, } \\
\text { hojas de trabajo, mapas de la comunidad }\end{array}$ \\
\hline 2 & $\begin{array}{l}\text { Identificar sitios y eventos dentro de esas áreas } \\
\text { donde las personas acuden para conocer a nuevas } \\
\text { parejas sexuales }\end{array}$ & $\begin{array}{l}\text { Encuestas aplicadas a informantes claves de la } \\
\text { comunidad }\end{array}$ & $\begin{array}{l}\text { Lista única de sitios donde las personas van a conocer } \\
\text { a nuevas parejas sexuales }\end{array}$ \\
\hline 3 & $\begin{array}{l}\text { Visitar y caracterizar los sitios reportados por los } \\
\text { informantes claves de la comunidad }\end{array}$ & $\begin{array}{l}\text { Visitas a los sitios reportados para verificar su } \\
\text { existencia, ubicación y obtener información de sus } \\
\text { características mediante encuestas a un informante } \\
\text { clave del sitio }\end{array}$ & $\begin{array}{l}\text { C.uadro con las características de los sitios visitados, } \\
\text { características de las personas encuestadas y de los } \\
\text { clientes, disponibilidad de condones y actividades } \\
\text { preventivas sobre ITS/VIH/SIDA }\end{array}$ \\
\hline 4 & $\begin{array}{l}\text { Identificar características de las personas que } \\
\text { socializan o que interactúan en los sitios }\end{array}$ & $\begin{array}{l}\text { Encuestas breves a clientes de una muestra de } \\
\text { personas que socializaban en los sitios }\end{array}$ & $\begin{array}{l}\text { C.uadro con características de los clientes, frecuencia } \\
\text { de visitas a los sitios, número de nuevas parejas } \\
\text { sexuales, uso de preservativos, etcétera }\end{array}$ \\
\hline & Resumir resultados y selección de indicadores & A nálisis estadístico & $\begin{array}{l}\text { R.eporte de resultados que incluyen los indicadores } \\
\text { basales para monitoreo futuro }\end{array}$ \\
\hline
\end{tabular}

\section{Visita y caracterización de cada sitio}

En Chetumal, durante esta etapa de seis días, 10 encuestadores visitaron 134 sitios para comprobar su existencia, caracterizarlos y encuestar a un ICS; se localizaron 101 sitios (75.3\%). El 10\% rechazó participar. De los lugares visitados y caracterizados en 89 sitios se encuestó a un ICS; el promedio de edad de los encuestados fue de 33 años. El 22\% fueron mujeres. Más de la $\operatorname{mitad}(52.6 \%)$ de los sitios fueron bares/cantinas o restaurantes (cuadro III). En Ciudad Hidalgo tres encuestadores, durante 14 días, visitaron 42 sitios en los cuales se encuestó a un ICS; el promedio de edad de los encuestados fue de 35 años. El 57.7\% fueron mujeres. El $55 \%$ de los sitios fueron bares o restaurantes. Las botaneras (establecimientos en donde sirven bebidas alcohólicas acompañadas de alimentos) representaron 12\% de los sitios (cuadro III). Sólo en esta área de estudio fueron encontrados casas particulares usados como casas de citas o de encuentro en donde una pareja previamente conocida puede ir para tener relaciones sexuales.

En Chetumal 53\% de los ICS encuestados en el lugar confirmaron que la gente conoce a nuevas parejas sexuales, $51 \%$ reportó que los hombres, y $48.3 \%$ que las mujeres conocen a nuevas parejas y al menos 17\% mencionó que los hombres conocen allí a parejas masculinas. Cerca de 17\% de los encuestados estima que al menos la mitad de los hombres que llegan al sitio conocen a una nueva pareja sexual, en comparación con sólo 10\%, que opina lo mismo de las mujeres. En Ciudad Hidalgo al menos 60\% de los ICS encuesta- dos reportaron que las personas conocen ahí nuevas parejas sexuales. El 54.8\% reportó que los hombres y $47.6 \%$ que las mujeres conocen a nuevas parejas; al menos 19\% mencionó que los hombres conocen a nuevas parejas masculinas en el sitio. El 22\% de las personas encuestadas reportó que al menos la mitad de los hombres conocen a nuevas parejas mientras permanecen en el sitio, en comparación con $26 \%$, que reportó lo mismo para las mujeres.

De los lugares visitados en Chetumal en más de $70 \%$ se reportó la presencia de hombres y mujeres que viajan a través del área regularmente o que sólo están de paso. Los ICS reportaron tanto población móvil como la población local que ahí llega; la proporción fue de $66 \%$ para el caso de hombres, y 59\% para las mujeres usuarias. En Ciudad Hidalgo, aproximadamente en 88\% de los sitios, se reportó población móvil, hombres y mujeres que llegan al sitio; y en cuanto a hombres y mujeres residentes en ambas ciudades, su presencia en los sitios de encuentro fue reconocida en 76.2 y $69 \%$ de ellos, respectivamente. Tanto la población móvil como la población local interactúan en aproximadamente tres cuartas partes de estos lugares.

En Chetumal, los hombres migrantes y los trabajadores agrícolas socializan en poco más de $40 \%$ de los sitios; las trabajadoras migrantes visitan $28.1 \%$ y las trabajadoras agrícolas acuden a 19\% de estos lugares. En Ciudad Hidalgo se reportó, en casi dos tercios de los sitios, la asistencia de trabajadores migrantes y $66.7 \%$ reportó a mujeres trabajadoras migrantes.

En el área de Chetumal los encuestados reportaron que los traileros o taxistas acuden a $63 \%$ de los si- 


\section{Cuadro III \\ Características de los Sitios visitados. MÉxıco, 2001}

\begin{tabular}{|c|c|c|}
\hline Característica & $\begin{array}{c}\text { Chetumal } \\
\text { Q uintana Roo } \\
\%\end{array}$ & $\begin{array}{c}\text { Ciudad Hidalgo } \\
\text { Chiapas } \\
\%\end{array}$ \\
\hline Tipo de sitios & $(n=89)$ & $(n=42)$ \\
\hline Restaurante & 17.98 & 26.19 \\
\hline Discoteca & 7.87 & 2.38 \\
\hline Bar /cantina & 34.83 & 28.57 \\
\hline Sitio con table dance & 5.62 & 0 \\
\hline Hotel & 5.62 & 7.14 \\
\hline Parque / Plaza & 7.87 & 7.14 \\
\hline Una calle & 4.49 & 2.38 \\
\hline Botanera & 3.37 & 11.90 \\
\hline Iglesia & 1.12 & 0 \\
\hline Estación de autobuses & 2.25 & 0 \\
\hline 0 tros & 8.99 & 14.29 \\
\hline Total & 100 & 100 \\
\hline \multicolumn{3}{|l|}{ Actividades para la prevención delVIH/SIDA } \\
\hline en el sitio & $(n=89)$ & $(n=42)$ \\
\hline Cualquier cartel sobre VIH/SIDA & 0 & 9.52 \\
\hline Sin información & 12.36 & 0 \\
\hline Folletos sobreVIH/SIDA & 0 & 7.14 \\
\hline Sin información & 12.36 & 0 \\
\hline Disponibilidad del condón en el sitio & $(n=89)$ & $(n=42)$ \\
\hline Condones en el sitio en el momento de la visita & 4.49 & 9.52 \\
\hline Sin información & 12.36 & 0 \\
\hline Posibilidad de comprar condones dentro de & & \\
\hline 10 minutos después de dejar el sitio por la noche & 68.54 & 59.52 \\
\hline Sin información & 6.74 & 0 \\
\hline \multicolumn{3}{|l|}{ Frecuencia de disponibilidad del condón } \\
\hline en el año pasado & $(n=89)$ & $(n=42)$ \\
\hline Condones siempre disponibles aquí & & \\
\hline en el año pasado & 3.37 & 26.19 \\
\hline \multicolumn{3}{|l|}{ Condones disponibles aquí a veces } \\
\hline en el año pasado & 10.11 & 4.76 \\
\hline Sin información & 7.87 & 0 \\
\hline Presencia de condones en el sitio el día de la visita & $(n=89)$ & $(n=42)$ \\
\hline Sí hay condones, pero no fueron vistos & 2.25 & 7.14 \\
\hline No hay condones & 76.40 & 71.43 \\
\hline Sin información & 14.61 & 0 \\
\hline Actividades en los sitios & $(n=75)$ & $(n=38)$ \\
\hline Se consume cerveza & 80 & 81.58 \\
\hline Sin información & 0 & 0 \\
\hline Se consume licor & 76 & 21.05 \\
\hline Sin información & 0 & 0 \\
\hline Formación de parejas & $(n=89)$ & $(n=42)$ \\
\hline Una persona en el sitio facilita el & 4.49 & 4.76 \\
\hline Sin información & 10.11 & 0 \\
\hline TSC buscan clientes en ese sitio & 17.98 & 21.43 \\
\hline Sin información & 11.24 & 0 \\
\hline Las personas que se conocen en el sitio & & \\
\hline tienen relaciones sexuales en el local & 5.62 & 19.05 \\
\hline Sin información & 19.10 & 4.76 \\
\hline Las personas que se conocen en el sitio & & \\
\hline tienen relaciones sexuales en un hotel cercano & 26.97 & 40.48 \\
\hline Sin información & 31.46 & 0 \\
\hline
\end{tabular}

Fuente: encuesta a informante clave del sitio, Método PLACE, 2001 tios visitados, mientras que las mujeres trabajadoras del sexo comercial pueden ser localizadas en una tercera parte de los lugares donde se realizó la encuesta. Se informó la presencia de militares en $65 \%$ de los sitios. En Ciudad Hidalgo, en $86 \%$ de los sitios, los trabajadores del transporte acuden al lugar y en $45 \%$ se indicó que las trabajadoras del sexo comercial llegan ahí. Los militares pueden ser identificados en $45 \%$ de los sitios. No se reportó la asistencia o visita exclusiva de algún tipo de usuario o cliente específico en ninguna de las dos ciudades.

En Chetumal, en aproximadamente 20\% de los sitios, los ICS reportaron que los empleados, hombres y mujeres conocen a nuevas parejas en el lugar. Los encuestados reportaron a hombres menores de 18 años de edad en $54 \%$ de los sitios, y a mujeres menores de edad en $51 \%$ de ellos. En Ciudad Hidalgo, en $14.3 \%$ de los establecimientos, se reportó que sus empleados conocen a nuevas parejas sexuales y en $30.9 \%$ se reportó lo mismo para las empleadas. En 14.6\% de los lugares se reportó a los hombres menores de edad y en $26 \%$ la presencia de mujeres menores de 18 años de edad.

Respecto a la disponibilidad de condones al interior de los sitios, en Chetumal, en sólo 6.7\%, hubo condones que fueron vistos en el momento de la encuesta. Cerca de $78.6 \%$ de los sitios nunca habían tenido condones durante los 12 meses previos (cuadro III), sin embargo, en $84 \%$ de los sitios la persona responsable encuestada estuvo dispuesta a vender o permitir la distribución de condones al interior del lugar. El 15.7\% de los sitios ha tenido alguna vez actividades de prevención de ITS/ $\mathrm{VIH} / \mathrm{SIDA}$, y en 94\% de ellos los responsables estuvieron dispuestos a permitir actividades de prevención. En Ciudad Hidalgo se reportó que en $21.4 \%$ de los sitios los condones estaban disponibles y fueron vistos. Según los encuestados en $69 \%$ de los sitios nunca hubo condones el año anterior a la fecha del estudio, tampoco actividades de prevención de ITS/VIH/SIDA (cuadro III). El 93\% de los encuestados estuvieron de acuerdo en tener actividades de prevención, y 91\% aceptó apoyar la venta o distribución de condones al interior del lugar.

\section{Encuestas con las personas que interactuaban en los sitios}

En Chetumal, en 39 sitios se contactó para encuestar a 709 individuos que interactuaban; aproximadamente, dos tercios de los encuestados fueron hombres. Del total $11 \%$ rechazó participar, de lo que se obtuvo un total de 628 cuestionarios. El promedio de edad de los hombres fue de 30 años y de 26 para las mujeres; al menos $54 \%$ de las mujeres fueron menores de 25 años. En Ciudad Hidalgo, las encuestas a individuos que 
interactuaban se realizaron en 41 sitios en donde se contactó a 263 personas, 13\% rechazó participar; al final, 229 personas fueron encuestadas. El 70\% de encuestados fueron hombres. El promedio de edad para los hombres fue de 32 años y 28 para mujeres, al menos $40 \%$ fueron menores de 25 años (cuadro IV).

En Chetumal se reportó que 32\% de los hombres y $40 \%$ de las mujeres encuestadas en los sitios acudieron al lugar de la encuesta al menos una vez a la semana durante las últimas cuatro. Entre las personas que dijeron no vivir en Chetumal, cerca de $37 \%$ de los hombres y $43 \%$ de mujeres dijeron visitar el sitio cada vez que ellos acuden a la ciudad. De las personas encuestadas en Ciudad Hidalgo 32\% de los hombres y $62 \%$ de las mujeres reconocieron haber visitado el sitio al menos una vez por semana en las cuatro semanas previas. Más de $30 \%$ de hombres y cerca de $60 \%$ de mujeres que viven fuera de la ciudad acuden al sitio de la encuesta cuando se encuentran en Ciudad Hidalgo.

En cuanto a la frecuencia de adquisición de nuevas parejas sexuales de las personas que socializaban en los sitios, cerca de $21 \%$ de las encuestadas en Chetumal y Ciudad Hidalgo informaron haber conocido una nueva pareja sexual en el sitio en alguna ocasión, la mayoría en las cuatro semanas previas al estudio. En Chetumal los hombres tuvieron en promedio 1.3 parejas en las cuatro semanas previas, de las cuales, 0.7 fueron nuevas. Las mujeres reportaron un promedio de 2.3 parejas sexuales en las últimas cuatro semanas; en promedio 1.8 fueron nuevas (cuadro IV). En Ciudad Hidalgo los hombres tuvieron en promedio una pareja sexual en las cuatro semanas previas, en promedio 0.4 fueron nuevas. Por otro lado, en las cuatro semanas previas al estudio las mujeres tuvieron un promedio de 2.9 parejas sexuales, en promedio, dos fueron nuevas. El 13\% de las mujeres comunicó haber tenido 10 o más parejas en el mismo periodo de referencia. Tanto en Chetumal como en Ciudad Hidalgo al menos 15\% de las mujeres reportaron haber tenido 10 o más parejas sexuales durante los 12 meses previos al estudio, $y$ de éstas, al menos 13\% fueron nuevas.

En Chetumal tres cuartas partes de los hombres encuestados han usado alguna vez un condón, en comparación con sólo la mitad de las mujeres encuestadas (cuadro IV). De las personas que tuvieron una nueva pareja sexual en las cuatro semanas previas al estudio, $60 \%$ informó haber usado un condón la última vez que tuvo relaciones con una nueva pareja. De las personas que han encontrado una nueva pareja sexual en el sitio, $57 \%$ usó un condón en la última relación con ésta. En Ciudad Hidalgo 70\% de los hombres han usado alguna vez un condón, en comparación con $51 \%$ de las mujeres. De las personas que tuvieron una nueva pareja sexual en las cuatro semanas previas al estudio $80 \%$ de los hombres y $93 \%$ de las mujeres reportaron haber usado condón la última vez que tuvieron relaciones sexuales con esta nueva pareja. De las personas que han encontrado una nueva pareja sexual en el sitio, $71 \%$ de hombres y mujeres encuestados usaron un condón en su última relación con ella.

El 20\% de todos los individuos que interactuaban en los sitios de Chetumal reportó emplear actividades de sexo transaccional durante las últimas cuatro semanas. Más de $20 \%$ de los hombres dieron dinero, regalos o favores a cambio de sexo, mientras que casi $24 \%$ de las mujeres recibieron estas cosas por sexo. En Ciudad Hidalgo se reportó que $27 \%$ de los hombres dieron y $26 \%$ de las mujeres recibieron dinero, además de regalos o favores a cambio de sexo en las cuatro semanas anteriores.

En cuanto a la presencia de signos o síntomas de alguna ITS en las cuatro semanas previas al estudio en las personas que se encontraban interactuando al interior de los sitios, en Chetumal $6.9 \%$ de los hombres reportaron "dolor al orinar", "flujo poco común" 3.9\% y "llagas o úlceras genitales" $1.4 \%$. El 29.7\% acudió para tratamiento médico. En el caso de las mujeres $15.8 \%$ reportó "dolor en la parte baja del abdomen", $14.2 \%$ "flujo poco común" y $4.20 \%$ "llagas o úlceras genitales". El $64.1 \%$ reportó que acudió por tratamiento médico. Por otra parte, en Ciudad Hidalgo, 5.1\% de los hombres reportaron "dolor al orinar", 3.8\% "flujo poco común" y $1.9 \%$ "llagas o úlceras genitales". El 10.4\% de las mujeres reportó "dolor en la parte baja del abdomen" y 2.9\% "flujo poco común". El 100\%, tanto de hombres como de mujeres, buscaron atención médica.

\section{Discusión}

La adaptación e implantación del protocolo de PLACE fue factible en las dos ciudades de la frontera sur de México. El estudio tiene la limitante de que se realizó en un periodo corto $\mathrm{y}$, por tanto, los datos pueden no reflejar las variaciones de movilidad poblacional a lo largo del año. Si bien la metodología empleada de los informantes claves identificó a la gran mayoría de los sitios específicos en donde los individuos conocen nuevas parejas, no es capaz de identificar todos, ya que los ICS reportaron sitios de encuentro (dos bares) diferentes a los reportados por los ICC que se abordaron en sitios públicos de las comunidades. Posiblemente los ICS tienen acceso a información sobre sitios de encuentro privados no tan accesibles a los ICC. Se observa que la venta de bebidas alcohólicas es la actividad predominante en estos lugares. Aunque pocos sitios tuvieron condones disponibles o han tenido programas de pre- 


\section{Cuadro IV \\ Características de las personas que socializan en los sitios seleccionados. México, 2001}

\begin{tabular}{|c|c|c|c|c|}
\hline \multirow[b]{2}{*}{ Característica } & \multicolumn{2}{|c|}{ C hetumal, Q uintana Roo } & \multicolumn{2}{|c|}{ Ciudad Hidalgo, Chiapas } \\
\hline & $\begin{array}{c}\text { Hombres } \\
\%\end{array}$ & $\begin{array}{c}\text { Mujeres } \\
\%\end{array}$ & $\begin{array}{c}\text { Hombres } \\
\%\end{array}$ & $\begin{array}{c}\text { Mujeres } \\
\%\end{array}$ \\
\hline Grupos de edad de las personas entrevistadas en el sitio & $(n=432)$ & $(n=196)$ & $(n=162)$ & $(n=67)$ \\
\hline $18-19$ & 12.27 & 15.82 & 8.02 & 13.43 \\
\hline $20-24$ & 22.69 & 38.78 & 24.69 & 26.87 \\
\hline $25-29$ & 24.31 & 21.94 & 17.90 & 31.34 \\
\hline $30-34$ & 13.66 & 12.76 & 16.67 & 11.94 \\
\hline $35-39$ & 10.19 & 5.10 & 14.81 & 5.97 \\
\hline $40-44$ & 5.79 & 2.55 & 3.70 & 4.48 \\
\hline $45-49$ & 4.86 & 1.02 & 6.17 & 1.49 \\
\hline $50-54$ & 3.01 & 0.51 & 4.32 & 2.99 \\
\hline 55 y más & 3.24 & 1.53 & 3.70 & 1.49 \\
\hline Total & 100 & 100 & 100 & 100 \\
\hline Domicilio actual & $(n=432)$ & $(n=196)$ & $(n=162)$ & $(n=67)$ \\
\hline Tiene su domicilio actual dentro del área de estudio & 41.90 & 43.88 & 40.74 & 26.87 \\
\hline Sin información & 0.23 & 0.51 & 1.23 & 1.49 \\
\hline Escolaridad (años de estudio) & $(n=432)$ & $(n=196)$ & $(n=162)$ & $(n=67)$ \\
\hline $\mathrm{N}$ inguno & 0.93 & 2.55 & 6.17 & 22.39 \\
\hline $1-6$ años (primaria) & 12.73 & 16.33 & 25.93 & 41.79 \\
\hline 7 - 9 años (secundaria) & 31.48 & 33.67 & 21.60 & 19.40 \\
\hline 10 - 12 años (bachillerato) & 28.47 & 20.92 & 25.93 & 10.45 \\
\hline$>12$ años (otros) & 26.39 & 25.51 & 19.75 & 5.97 \\
\hline Sin información & 0 & 1.02 & 0.62 & 0 \\
\hline Total & 100 & 100 & 100 & 100 \\
\hline Estado de empleo & $(n=432)$ & $(n=196)$ & $(n=162)$ & $(n=67)$ \\
\hline Empleado & 75 & 56.12 & 87.04 & 86.57 \\
\hline Desempleado & 25 & 42.35 & 11.11 & 13.43 \\
\hline Sin información & 0 & 1.53 & 1.85 & 0 \\
\hline Total & 100 & 100 & 100 & 100 \\
\hline A sistencia a sesiones educativas sobre VIH/SIDA. & $(n=432)$ & $(n=196)$ & $(n=162)$ & $(n=67)$ \\
\hline Ninguna & 77.08 & 67.86 & 53.70 & 56.72 \\
\hline $1-5$ & 22.22 & 27.04 & 38.89 & 37.31 \\
\hline $6-10$ & 0.46 & 3.57 & 5.56 & 2.99 \\
\hline Más de 10 & 0.23 & 1.53 & 1.85 & 2.99 \\
\hline Sin información & 0 & 0 & 0 & 0 \\
\hline Total & 100 & 100 & 100 & 100 \\
\hline Número de parejas sexuales en las últimas cuatro semanas & $(n=432)$ & $(n=196)$ & $(n=162)$ & $(n=67)$ \\
\hline N inguna & 39.81 & 49.49 & 36.42 & 56.72 \\
\hline 1 & 32.18 & 25 & 46.30 & 20.90 \\
\hline 2 & 13.66 & 8.16 & 9.26 & 2.99 \\
\hline 3 & 8.33 & 5.10 & 4.32 & 1.49 \\
\hline $4-9$ & 4.17 & 5.10 & 3.70 & 1.49 \\
\hline $10-14$ & 1.62 & 3.06 & 0 & 5.97 \\
\hline$>15$ & 0.23 & 4.08 & 0 & 8.96 \\
\hline Sin información & 0 & 0 & 0 & 1.49 \\
\hline Total & 100 & 100 & 100 & 100 \\
\hline N úmero de parejas sexuales nuevas en las últimas cuatro semanas & $(n=432)$ & $(n=196)$ & $(n=162)$ & $(n=67)$ \\
\hline $\mathrm{N}$ inguna & 65.05 & 72.96 & 78.40 & 76.12 \\
\hline 1 & 18.75 & 8.16 & 12.35 & 1.49 \\
\hline 2 & 9.03 & 4.08 & 4.94 & 2.99 \\
\hline 3 & 3.24 & 3.57 & 3.09 & 0 \\
\hline $4-9$ & 2.78 & 5.10 & 1.23 & 2.99 \\
\hline $10-14$ & 0.69 & 3.57 & 0 & 8.96 \\
\hline $15-64$ & 0.23 & 2.55 & 0 & 4.48 \\
\hline Sin información & 0.23 & 0 & 0 & 2.99 \\
\hline Total & 100 & 100 & 100 & 100 \\
\hline Número de parejas sexuales en los últimos 12 meses & $(n=432)$ & $(n=196)$ & $(n=162)$ & $(n=67)$ \\
\hline Ninguna & 21.76 & 40.31 & 32.72 & 52.24 \\
\hline 1 & 24.54 & 25.51 & 27.16 & 23.88 \\
\hline 2 & 17.82 & 5.61 & 9.26 & 5.97 \\
\hline
\end{tabular}




\begin{tabular}{|c|c|c|c|c|}
\hline & Cont & & & \\
\hline 3 & 8.33 & 3.06 & 6.17 & 1.49 \\
\hline $4-9$ & 20.14 & 10.20 & 11.73 & 0 \\
\hline $10-14$ & 3.01 & 1.53 & 6.17 & 2.99 \\
\hline $15-60$ & 4.17 & 8.16 & 6.17 & 5.97 \\
\hline Más de 60 & 0.23 & 5.61 & 0 & 7.46 \\
\hline Sin información & 0 & 0 & 0.62 & 0 \\
\hline Total & 100 & 100 & 100 & 100 \\
\hline N úmero de parejas sexuales nuevas en los últimos 12 meses & $(n=432)$ & $(n=196)$ & $(n=162)$ & $(n=67)$ \\
\hline $\mathrm{N}$ inguna & 40.97 & 62.76 & 58.64 & 73.13 \\
\hline 1 & 19.91 & 9.18 & 12.35 & 7.46 \\
\hline 2 & 12.04 & 7.14 & 8.64 & 1.49 \\
\hline 3 & 6.25 & 0.51 & 2.47 & 1.49 \\
\hline $4-9$ & 15.51 & 5.61 & 8.64 & 2.99 \\
\hline $10-14$ & 2.31 & 2.55 & 5.56 & 2.99 \\
\hline $15-60$ & 2.78 & 7.14 & 3.09 & 10.45 \\
\hline Más de 60 & 0.23 & 5.10 & 0 & 0 \\
\hline Sin información & 0 & 0 & 0.62 & 0 \\
\hline Total & 100 & 100 & 100 & 100 \\
\hline ¿Piensa usted que la gente viene aquí a conocer a nuevas parejas? & $(n=432)$ & $(n=196)$ & $(n=162)$ & $(n=67)$ \\
\hline Sí & 66.90 & 61.73 & 66.05 & 67.16 \\
\hline No & 31.94 & 37.24 & 33.95 & 31.34 \\
\hline Sin información & 1.16 & 1.02 & 0 & 1.49 \\
\hline Total & 100 & 100 & 100 & 100 \\
\hline ¿U sted ha encontrado una pareja aquí? & $(n=432)$ & $(n=196)$ & $(n=162)$ & $(n=67)$ \\
\hline Sí & 20.14 & 21.94 & 21.60 & 20.90 \\
\hline No & 79.86 & 78.06 & 77.78 & 79.10 \\
\hline Sin información & 0 & 0 & 0.62 & 0 \\
\hline Total & 100 & 100 & 100 & 100 \\
\hline ¿C uándo encontró a una pareja por última vez? & $(n=432)$ & $(n=196)$ & $(n=162)$ & $(n=67)$ \\
\hline En la última semana & 3.94 & 11.22 & 3.70 & 11.94 \\
\hline Hace 2-4 semanas & 5.56 & 6.12 & 6.79 & 2.99 \\
\hline Hace 2-6 meses & 5.09 & 0.51 & 5.56 & 0 \\
\hline Hace 7-12 meses & 1.16 & 0.51 & 0.62 & 1.49 \\
\hline Hace más de un año & 4.40 & 3.57 & 3.09 & 2.99 \\
\hline N unca & 79.86 & 78.06 & 77.78 & 79.10 \\
\hline Sin información & 0 & 0 & 2.47 & 1.49 \\
\hline Total & 100 & 100 & 100 & 100 \\
\hline ¿Dónde conoció a su pareja más reciente? & $(n=432)$ & $(n=196)$ & $(n=162)$ & $(n=67)$ \\
\hline En este sitio & 10.88 & 19.39 & 15.43 & 17.91 \\
\hline En otro lugar & 47.69 & 17.35 & 21.60 & 8.96 \\
\hline No nueva pareja sexual & 40.97 & 62.76 & 58.64 & 73.13 \\
\hline Sin información & 0.46 & 0.51 & 4.32 & 0 \\
\hline Total & 100 & 100 & 100 & 100 \\
\hline ¿Ha usado alguna vez un condón? & $(n=432)$ & $(n=196)$ & $(n=162)$ & $(n=67)$ \\
\hline Sí & 74.31 & 47.96 & 70.37 & 50.75 \\
\hline No & 25.69 & 48.98 & 29.63 & 49.25 \\
\hline Sin información & 0 & 3.06 & 0 & 0 \\
\hline Total & 100 & 100 & 100 & 100 \\
\hline ¿Trae un condón con usted? & $(n=432)$ & $(n=196)$ & $(n=162)$ & $(n=67)$ \\
\hline Sí trae y fue visto & 9.95 & 2.55 & 6.17 & 5.97 \\
\hline Sí trae pero no fue visto & 3.01 & 0.51 & 2.47 & 20.90 \\
\hline No trae condón & 87.04 & 96.86 & 91.36 & 71.64 \\
\hline Sin información & 0 & 4.08 & 0 & 1.49 \\
\hline Total & 100 & 100 & 100 & 100 \\
\hline ¿Usó un condón con su pareja más reciente de este lugar? & $(n=432)$ & ( $n=196)$ & $(n=162)$ & $(n=67)$ \\
\hline Sí & 11.57 & 12.76 & 15.43 & 14.93 \\
\hline No & 8.33 & 9.18 & 4.32 & 4.48 \\
\hline No pareja & 79.86 & 78.06 & 77.78 & 79.10 \\
\hline Sin información & 0.23 & 0 & 2.47 & 1.49 \\
\hline Total & 100 & 100 & 100 & 100 \\
\hline
\end{tabular}

Fuente: Encuesta a personas que socializaban en el sitio. Método PLACE 2001 
vención del VIH/SIDA en el lugar, en la mayoría de ellos estuvieron receptivos a las actividades de prevención como a la venta o distribución de condones al interior de sus locales, lo que sugiere un alto potencial para que éstos se conviertan en puntos de intervención. Esto permitiría llegar, no sólo a personas con un alto nivel de adquisición de nuevas parejas, si no también a las poblaciones móviles a las que es frecuentemente difícil abordar con mensajes sobre la prevención del VIH/ SIDA. Se observa que los ICS opinan que los usuarios conocen nuevas parejas sexuales y que éstos opinan lo mismo de otras personas, es decir, las otras personas usuarias del sitio sí conocen a nuevas parejas sexuales, pero al momento de cuestionarlas sobre si han conocido parejas sexuales, un porcentaje alto reportó que no lo hace. Los ICS reportaron la presencia de usuarios menores de edad, lo que se confirmó al observarlos en los sitios, pero por razones del diseño del estudio no fue posible incluirlos. Es importante obtener información respecto a conocimientos y prácticas sexuales en esta población dado el impacto de las ITS y el VIH/ SIDA en los jóvenes. Puesto que se observó que un grupo reducido de las mujeres tiene un número importante de nuevas parejas sexuales, se necesitan programas enfocados también a las parejas de estas mujeres. Llama la atención el autorreporte de la presencia de ITS en las personas que interactúan en los sitios, y la limitada búsqueda de atención médica en Chetumal, sin embargo los datos deben interpretarse con cuidado debido a que sólo se exploró la percepción del individuo. En general, en ambas áreas de estudio se observa que las mujeres que interactúan en los sitios, en comparación con los hombres, son más jóvenes, tienen menor escolaridad, una mayor proporción, están sin empleo, tienen más parejas sexuales y el uso de condones alguna vez es más bajo. Se reportaron actividades de sexo transaccional en ambas localidades; un mayor porcentaje fue reportado por las mujeres. Este fenómeno puede jugar un papel importante que alimenta en las mujeres la vulnerabilidad frente al VIH/ITS.

Se confirmó que la población local y móvil, las trabajadoras del sexo comercial, los jóvenes, incluyendo a menores de edad, interactúan en sitios específicos de las localidades, lo que abre una ventana de oportunidad para incidir con acciones preventivas adecuadas al contexto local de la infección por el VIH y demás ITS, aprovechando la disposición de los responsables o dueños de los sitios de implantar actividades de este tipo. Los sitios donde hay interacción entre estos grupos diferentes pueden ser puntos apropiados para reducir el comportamiento sexual de riesgo y aplicar otras intervenciones, con el fin de reducir su vulnerabilidad. Los resultados de los estudios de PLACE pueden ser im- portantes para la planificación estratégica regional de actividades de prevención del VIH en poblaciones móviles en México y Centroamérica. Algunos resultados son usados como indicadores de los programas nacionales de SIDA. ${ }^{12}$ En otros países el método PLACE mostró que es aceptable, flexible y no estigmatizante; y fue capaz de identificar áreas donde las personas con altas tasas de adquisición de nuevas parejas sexuales pueden ser alcanzadas con acciones de prevención. ${ }^{13}$

El estudio confirmó que Chetumal y Ciudad Hidalgo son áreas con necesidad de actividades para la prevención de ITS/VIH/SIDA, como facilitar la distribución de condones, promover la educación interpares y facilitar el acceso a la atención médica de las ITS.

\section{Referencias}

1. 0 rganización Panamericana de la Salud. Hacer frente al SIDA: prioridades de la acción pública ante una epidemia mundial.W ashington, DC: O PS, 1998; Publicación Científica N 0.570.

2.Yorke JA, Hethcote HW, N old A. Dynamics and control of the transmission of gonorrhea. Sex Transm D is 1978;5:51-57.

3. Garnett GP,Anderson RM. Sexually transmitted diseases and sexual behavior: Insights from mathematical models.J Infect $D$ is 1996:174

(Suppl 2):S150-S161.

4. W asserheit JN , Aral SO.The dynamic topology of sexually transmitted disease epidemics: Implications for prevention strategies.J Infect D is 1996;174(Suppl 2):S201-S213.

5.W eir S, Morroni C, C oetzee N, Spemcer J, Boerma T. A pilot study of a rapid assessment method to identify places for AID $S$ prevention in Cape Town, South A frica. Sex Transm Infect 2002; 70(Suppl I):i106-i113. 6. Bronfman M, Leyva-Flores R, N egroni M. Mobile populations and HIV/ AIDS in Central America, Mexico and the United States. En:XIV International AIDS Conference; 2002 July 7-12; Barcelona, España. Bologna (Italy): Monduzzi Editore, 2002129-134.D isponible en: http:// www.monduzzi.com/proceedings/moreinfo/20020708.htm.

7. Bronfman M, Sejenovich G, U ribe P. Migración y SIDA en México y América Central. México, D F: CO N A SIDA, Angulos del SIDA, 1998;

(2):21-31.

8. Instituto $\mathrm{N}$ acional de Estadística Geografía e Informática. Estados Unidos Mexicanos. XII Censo general de población y vivienda, 2000.

Disponible en www.inegi.gob.mx/.

9. Consejo $\mathrm{N}$ acional de Población. Conapo 2000. Disponible en www.conapo.gob.mx.

10. Consejo Nacional de Población. Concepto y dimensiones de la marginación. Conapo 2000. Disponible en: www.conapo.gob.mx/ m_en_cifras/marg2000/001.htm.

11. Secretaría de Salud, Dirección General de Epidemiología. Sistema único de Información y Vigilancia Epidemiológica (SUIVE). México, DF:SSA, 2001.

12. O N USIDA. Programa Conjunto de la N aciones Unidas sobre elVIH/ SIDA. Programas N acionales de SIDA. G uía para el Monitoreo y la Evaluación. Ginebra, Suiza: O N USIDA, 2000: 25-28.

13. Weir SS, BoermaT. From people to places: 0 verview of the PLACE Method and lessons learned. Measure Eval Bull 2002;4:1-6. Disponible en: http://www.cpc.unc.edu/measure/publications/bulletins/bulletin4/ bulletin4.html. 